\title{
Rose-Marie Arbour
}

PhD, histoire de l'art, professeure associée, Département d'histoire de l'art, UQÀM

(1993)

\section{"Une difficile interdisciplinarité dans l'enseignement des arts ou quand tous les ingrédients sont là pour ce faire mais que c'est toujours aussi difficile à réaliser...”}

\author{
Un document produit en version numérique par Jean-Marie Tremblay, bénévole, \\ professeur de sociologie au Cégep de Chicoutimi \\ Courriel: jean-marie tremblay@uqac.ca \\ Site web pédagogique : http://www.uqac.ca/jmt-sociologue/ \\ Dans le cadre de la collection: "Les classiques des sciences sociales" \\ Site web: http://classiques.uqac.ca/ \\ Une collection développée en collaboration avec la Bibliothèque \\ Paul-Émile-Boulet de l'Université du Québec à Chicoutimi \\ Site web: http://bibliotheque.uqac.ca/
}


Cette édition électronique a été réalisée par Jean-Marie Tremblay, bénévole, professeur de sociologie au Cégep de Chicoutimi à partir de :

\section{Rose-Marie Arbour}

[PhD, histoire de l'art, professeure associée, Département d'histoire de l'art, UQÀM.]

"Une difficile interdisciplinarité dans l'enseignement des arts ou quand tous les ingrédients sont là pour ce faire mais que c'est toujours aussi difficile à réaliser...”.

Communication au Séminaire Pédagogie et croisement des disciplines, Le Fresnoy, Tourcoing, (France), studio national des arts contemporains, 9 et 10 novembre 1993.

[Autorisation accordée le 31 décembre 2006 par Mme Arbour de diffuser toutes ses publications dans Les Classiques des sciences sociales.]

Courriel : arbour.rose-marie@uqam.ca

Polices de caractères utilisée :

Pour le texte: Times New Roman, 14 points.

Pour les citations : Times New Roman, 12 points.

Pour les notes de bas de page : Times New Roman, 12 points.

Édition électronique réalisée avec le traitement de textes Microsoft Word 2004 pour Macintosh.

Mise en page sur papier format : LETTRE (US letter), 8.5'” x 11'”)

Édition numérique réalisée le 10 mai 2007 à Chicoutimi, Ville de Saguenay, province de Québec, Canada. 


\section{Table des matières}

La formation de l'artiste à l'université

Formation universitaire/formation professionnelle en arts: une tension entre deux milieux

Interdisciplinarité et théorisation

Création disciplinaire

$\underline{\text { Pourquoi viser l'interdisciplinarité dans la formation universitaire des }}$ étudiants en arts? 
Rose-Marie Arbour

“Une difficile interdisciplinarité dans l'enseignement des arts ou quand tous les ingrédients sont là pour ce faire mais que c'est toujours aussi difficile à réaliser...”.

Communication au Séminaire Pédagogie et croisement des disciplines, Le Fresnoy, Tourcoing, (France), studio national des arts contemporains, 9 et 10 novembre 1993.

\section{La formation de l'artiste à l'université}

\section{$\underline{\text { Retour à la table des matières }}$}

D'une façon générale, si une définition de l'artiste se construit, au moins partiellement, à partir et en fonction de la pratique artistique où elle s'exerce, quand elle se situe, et de sa fonction actuelle dans la société, le corrélat est de cerner le type de formation que doit recevoir un étudiant en arts à l'université.

La formation de l'artiste entre dans la mission première de l'UQAM, université montréalaise créée en 1969 à partir de diverses écoles existantes, dont l'Ecole des Beaux-Arts, une école de musique, une section d'art dramatique.

Le Secteur des Arts de l'UQAM comprend sept programmes de Bacc spécialisé en arts (arts visuels, histoire de l'art, design graphique, design de l'environnement et les programmes en art d'interprétation: musique, danse et art dramatique). (=Licence française) et cinq programme de M.A (arts plastiques, danse, théâtre, études des arts, muséologie). 
La population étudiante inscrite au niveau des Bacc est de 2,383 étudiants (1,515 t.c., 868 t.p.) Le cours Atelier interdisciplinaire en arts s'adresse donc à une minorité d'étudiants de diverses disciplines artistiques inscrits dans ces programmes; la majorité d'entre eux cependant venait d'arts visuels.

Une expérience pédagogique interdisciplinaire a été réalisée depuis quatre ans au Secteur des Arts de l'UQAM - dont les deux dernières (1992 et 1993) ont été assumées par Alain Fleischer dans un cours intitulé Atelier interdisciplinaire en arts (90 hres/session). Ce cours a été créé officiellement et pourvu d'un budget annuel intéressant depuis deux ans. Le niveau académique des étudiants se situait à la troisième année du Bacc spécialisé en arts (équivalent de la Licence française).

Je vous expose ici le contexte académique du Secteur des Arts de l'UQAM pour bien situer les enjeux et les difficultés concrètes que soulève la question de l'interdisciplinarité dans les programmes d'art à l'université: les difficultés surgissent autant des structures académiques et administratives que de la résistance des mentalités face à la transgression des frontières.

\section{Formation universitaire/formation professionnelle en arts: une tension entre deux milieux}

$\underline{\text { Retour à la table des matières }}$

La formation en arts est tiraillée, faut-il le souligner, entre les normes de formation universitaire proprement dites et des normes de formation professionnelle émanant de divers milieux de travail et de pratiques. 
L'enseignement universitaire vise une formation théorique et une formation pratique qui consistent à rendre apte l'étudiant à analyser et à comprendre les fondements de sa pratique disciplinaire ainsi que les conditions théoriques, historiques, sociales et économiques qui ont constitué sa discipline. Son objectif est l'apprentissage d'un savoir spécialisé et de savoirs-faire dans le cadre d'une discipline donnée tout en favorisant des apports complémentaires venant des domaines des communications, des sciences humaines et des sciences de l'éducation particulièrement.

Cette double formation vise à favoriser une capacité critique qui incite l'étudiant à s'engager dans des démarches exploratoires, sans lesquelles un processus de création ne peut être envisagé.

Une tension réelle existe entre cette formation théorie/pratique et les exigences d'une formation professionnelle auxquelles l'enseignement doit également répondre.

Les normes et les comportements professionnels sont définis par les milieux externes - corporations, groupes constitués, pratiques aux usages plus ou moins officialisés, milieux de diffusion/réception, usage de plus en plus généralisé de nouvelles technologies etc....

L'université ne peut ignorer les incitatifs d'efficacité et de productivité de même que les exigences techniques venant de ces milieux externes. Mais elle doit se démarquer des écoles d'art professionnelles et des conservatoires: Il est bien connu que la formation donnée par exemple dans les conservatoires de musique, de théâtre, de danse, est repliée à l'intérieur de frontières disciplinaires strictes et exclusives.

À l'UQÀM, de plus, la notion de profession et la notion de discipline sont loin d'être les mêmes pour chacun des sept programmes de Bacc. Les exigences des milieux externes, selon les disciplines, sont plus ou moins précises et plus ou moins grandes. L'horizon d'attente 
par rapport à l'ensemble de ces disciplines enseignées est donc des plus hétéroclites.

Enfin, les pressions des divers milieux qui s'affrontent dans le champs artistique, les incitations des institutions subventionnaires qui sont particulièrement présentes au Canada et au Québec, celles vouées à la diffusion de l'art contemporain et actuel, représentent d'autres contraintes - sans parler de la nécessité d'intégrer les nouvelles technologies dans ces domaines de savoirs et de pratiques.

En principe, cette formation universitaire vise également à formaliser des liens (réels ou virtuels) entre les disciplines artistiques ellesmêmes et entre ces dernières et d'autres champs de savoirs et d'habiletés, afin de stimuler et d'élargir le domaine de la création artistique. C'est du moins l'idéal visé mais cela est loin de se concrétiser dans les faits.

Inscrire la notion d'interdisciplinarité en arts dans un tel contexte académique et professionnel n'est pas simple - même si à l'UQÀM tous les programmes profitent d'installations matérielles (ateliers, studios en audio-visuel, informatique, salles de répétition, ateliers de fabrication de costumes etc) qui sont placées les unes à proximité des autres mais qui sont néanmoins le fief de chacune des disciplines actuellement enseignées.

On peut se demander - dès lors que sont objectivement réunies les conditions matérielles et les ressources professorales pour favoriser et stimuler les échanges multi et interdisciplinaires, et que des ressources relativement importantes existent, - comment il se fait que les tentatives pour insérer l'interdisciplinarité dans les programmes aient été jusqu'à maintenant des plus timides et qu'aucun programme n'affiche, dans son bloc de cours obligatoires ou même optionnels, des pratiques interdisciplinaires qui dépassent la seule notion de multidimensionnalité propre à certaines de ces disciplines? 


\section{Interdisciplinarité et théorisation}

$\underline{\text { Retour à la table des matières }}$

En fait on constate que très peu d'institutions de formation universitaire sont aptes à intégrer le phénomène de l'interdisciplinarité en partie du fait qu'il remet en question les bases mêmes des structures et de la philosophie d'une formation traditionnelle en $\operatorname{arts}^{1}$ et des structures institutionnelles fondées sur les départements disciplinaires..

Dans les domaines scientifiques (sciences sociales, sciences biologiques...) elle signifie et est le résultat d'une synthèse habituellement réalisée par des équipes de spécialistes.

En arts, la situation est différente: l'art est moins un champ de connaissance qu'un domaine de pratique et c'est pourquoi la conceptualisation et, par là, l'enseignement interdisciplinaire, sont en dans une position difficile.

L'interdisciplinarité n'étant pas véritablement définie, il est difficile de l'enseigner, de l'aborder théoriquement - d'où l'entre-deux où se situent les artistes-professeurs qui s'y engagent.

De plus, dans la situation actuelle les professeurs et les étudiants hésitent à consacrer des cours à des activités qui ne viennent pas directement consolider leur discipline respective - surtout dans les arts d'interprétation où la dimension performative est très importante. Une autre difficulté s'ajoute à celle-là et qui n'est pas négligeable: les cours

1 Je me suis inspirée en partie du texte publié par Danielle Boutet, "Interdisciplinarity in the Arts", Harbour, Magazine of Art \& Everyday Life, no. 6, 1993. pp. 66-72 
interdisciplinaires regroupent des étudiants de formations diverses, ce qui crée forcément des décalages entre eux au sein de ces cours - ces décalages se transformant souvent en inégalités.

C'est donc partiellement à cause du manque de théorisation que l'interdisciplinarité en arts n'est pratiquement pas enseignée sauf dans des expériences ponctuelles

et

elle est difficile à enseigner si on veut le faire dans les termes des disciplines connues i.e. en termes de procédures et de techniques seulement.

\section{Création disciplinaire}

$\underline{\text { Retour à la table des matières }}$

Les processus qui mènent à la réalisation d'une peinture, à la création d'une chorégraphie, sont compris, conceptualisés.

Ce qui n'est pas le cas quand il s'agit d'un projet interdisciplinaire où ils ne sont la plupart du temps abordés en début de projet que sous l'angle esthétique et historique.

L'enseignement traditionnel des arts (les spécialités) consiste généralement à enseigner des techniques et des traditions esthétiques; l'étudiant est laissé à lui-même quant à sa "créativité" qui devra se muer en "création" grâce au passage qui le mènera à la réalisation d'une oeuvre - dans un tel contexte, le mythe de la création et la magie de l'intuition créatrice occupent une position dominante.

L'atelier interdisciplinaire met au centre d'intérêt et de préoccupation le processus créateur, justement parce qu'il s'élabore en dehors du contexte connu des disciplines traditionnelles. Le processus créateur 
est à constituer par l'étudiant ou l'artiste interdisciplinaire, par le choix conscient de procédures, la manipulation de techniques, l'utilisation de méthodes, de médiums, la définition de buts et d'objectifs, l'usage d'outils, l'acquisition de certaines habiletés etc... L'intuition y perd son sens mythique et privé car l'oeuvre interdisciplinaire devient plutôt le résultat d'une mise en relation d'éléments divers, compris dans leurs caractéristiques spécifiques, mais qui fonctionnent dorénavant dans un autre contexte, un contexte nouveau qui ne peut pas exister dans les pratiques disciplinaires où il est déjà entièrement fixé avec les procédures, actions et techniques qui leur sont propres.

Les actions et procédures des étudiants qui travaillent à une entreprise interdisciplinaire, dès lors qu'elles sont connues et objectivées, peuvent être prises en charge par l'enseignement. (Il ne s'agit pas ici seulement de techniques qui, en ce qui concerne les nouvelles technologies, évoluent d'ailleurs extrêmement rapidement.)

Les étudiants qui travaillent avec des médiums et dans des situations non traditionnels, doivent donc créer leur théorie à mesure qu'ils créent leur propre contexte de conceptualisation et de production (procédures, étapes, concepts etc...). C'est pourquoi ces cours constituent de nouveaux contextes générateurs de théorie - et la théorie est essentielle pour envisager une formation universitaire.

Dans ce cours il faut donc y aménager des périodes de retour critique, d'analyse et de réflexion qui permettent d'objectiver les étapes franchies, les actions et transferts réalisés. De plus il faut prévoir en début de cours, des cours-catastrophes (Crash-Course) qui initient les étudiants qui en ont besoin à des outils et processus qui ne leur sont pas familiers. Ces deux volets différencient encore davantage un cours interdisciplinaire d'un cours disciplinaire.

Dans le contexte actuel de l'enseignement des arts une des questions qui ne peut être évitée est: quelle dynamique va s'instaurer entre 
l'enseignement disciplinaire traditionnel et l'enseignement interdisciplinaire?

\section{Pourquoi viser l'interdisciplinarité dans la formation universitaire des étudiants en arts?}

$\underline{\text { Retour à la table des matières }}$

Autant des outils matériels et conceptuels venant d'autres disciplines sont constamment susceptibles d'être utilisés en arts, autant il faut rappeler que les disciplines telles qu'elles existent actuellement ont été modelées par l'apport de disciplines diverses à l'origine.

Dans une entreprise interdisciplinaire, l'étudiant est amené à percevoir différemment l'espace et le temps, à réfléchir aux limites des disciplines, aux lieux possibles de leur ré-ouverture.

Savoir minimalement se servir d'un appareil vidéo pour un sculpteur, savoir aborder des questions de mise en scène par un peintre ou un designer, entraînent ultérieurement des modifications inédites dans la façon de se servir de leurs propres outils et méthodes conceptuels et techniques.

Il ne s'agit pas ici de viser la maîtrise des données, méthodes et processus d'une discipline autre: il s'agit plutôt d'un transfert qui va au-delà de la seule prise de conscience qu'il existe d'autres modalités de perception et d'appréhension de la réalité, d'autres processus disciplinaires. Ainsi, l'expérience menée avec Alain Fleischer à Montréal a mis l'accent sur la participation de l'étudiant à une équipe multidisciplinaire pour réaliser un projet qui lui, dans sa réalisation même, a été interdisciplinaire. Il va en parler lui-même. 
Pour ne parler que du domaine des arts, la formation actuelle des étudiants devrait se faire en regard non seulement de traditions à transmettre mais en regard des conditions complexes et nouvelles d'exercice de la création artistique: démantèlement des frontières entre les arts et les disciplines en général, hybridité, déstabilisation des certitudes et des savoir-faire au profit des emprunts de toutes sortes, remise à vif des relations entre arts, identités, territoires, remise en question des notions traditionnelles de qualité, de beauté, d'harmonie, d'unité d'expression et finalement de sens.

Les mentalités et les concepts, les références et les méthodes sont provoquées sans cesse par la télévision, l'interactivité, les outils électroniques sophistiqués, la réalité virtuelle. Tout cela ne peut plus être considéré comme éléments secondaires pour penser la formation des étudiants en arts.

En fin de piste, c'est la modification du processus créateur traditionnel qui a été structuré par et autour d'une discipline donnée, qui est ici envisagée par l'interdisciplinarité.

Fin du texte 